\title{
Amygdala $\beta$-Noradrenergic Receptors Modulate Delayed Downregulation of Dopamine Activity following Restraint
}

\author{
Chun-hui Chang and Anthony A. Grace \\ Departments of Neuroscience, Psychology, and Psychiatry, University of Pittsburgh, Pittsburgh, Pennsylvania 15260
}

\begin{abstract}
Stress, which involves a heightened arousal and excitability, triggers important adaptive responses to maintain homeostasis and prepare a response. In the current studies, we administered a psychological stressor of $2 \mathrm{~h}$ acute restraint on rats, and found that $24 \mathrm{~h}$ after the cessation of the restraint session, there was a significant decrease in ventral tegmental area dopaminergic (DA) neuron population activity and a significant attenuation in amphetamine-induced locomotor activity. Systemic or intra-basolateral nuclei of the amygdala administration of the $\beta$-noradrenergic receptor antagonist, propranolol, reversed the decrease, suggesting that the delayed attenuation of DA neuron firing following a stressor depends on a noradrenaline-mediated mechanism. This alteration in DA activity may adaptively prepare the individual to avoid the stressor, or in the extreme, may be a factor that contributes to pathological changes in behavior or physiological responses.
\end{abstract}

\section{Introduction}

Stress is defined as any threat, either real or perceived, to the homeostasis and well being of an organism (Herman and Cullinan, 1997; Sawchenko et al., 2000; Morilak et al., 2005; Joëls and Baram, 2009) and which may cause the subject to initiate appropriate responses to adapt to the changing environment (de Kloet et al., 2005; McEwen, 2007; Ulrich-Lai and Herman, 2009). The temporal profiles of stress responses span from milliseconds to days (Joëls and Baram, 2009). The rapid actions provoke vigilance and alertness of the situation for an optimal strategy to face the challenge, while the late actions promote the sustained and adaptive components of stress responses. Thus, how fast a stress response can be evoked and how long it lasts is critical to the physiological homeostasis and adaptation of the individual.

Previously, we found that $2 \mathrm{~h}$ acute restraint significantly increased ventral tegmental area (VTA) dopaminergic (DA) system responsivity when tested soon after the stressful event (Valenti et al., 2011). However, given that the effects of stressors change over time, it was not known whether this effect was maintained or was a transitional period preceding subsequent alterations, such as those that occur in the opponent-process model (Solomon and Corbit, 1974; Koob et al., 1997). Second, given the known involvement of the noradrenergic system in stress responses (Morilak et al., 2005), how the noradrenergic system impacted the stress-induced changes in VTA DA neuron activity was of interest both with respect to mechanisms and potential therapeutic inter-

\footnotetext{
Received May 19, 2012; revised Nov. 8, 2012; accepted Nov. 16, 2012.

Author contributions: C.-h.C. and A.A.G. designed research; C.-h.C. performed research; C.-h.C. analyzed data; C.-h.C. and A.A.G. wrote the paper.

This work was supported by United States Public Health Service Grant DA15408 (A.A.G.). We thank Niki MacMurdo, Dr. Linda Rinaman, and Vicki Maldovan for their valuable advice and technical assistance.

The authors declare no competing financial interests.

Correspondence should be addressed to Dr. Chun-hui Chang, Department of Neuroscience, University of Pittsburgh, A210 Langley Hall, Pittsburgh, PA 15260. E-mail: changch@pitt.edu.

DOI:10.1523/JNEUROSCI.2420-12.2013

Copyright $\odot 2013$ the authors $\quad 0270-6474 / 13 / 331441-10 \$ 15.00 / 0$
}

ventions. Noradrenergic neurons in the locus ceruleus (LC) are activated by stressful stimuli (Rasmussen et al., 1986; AstonJones et al., 1991; Morilak et al., 2005). The LC projects diffusely onto many forebrain nuclei (Jones and Moore, 1977) that are major targets relevant to stress, including basolateral nuclei of the amygdala (BLA; composed of the lateral, basal, and accessory basal nuclei) (LeDoux, 2000), which provides feedback to the LC via reciprocal projections (Van Bockstaele et al., 2001; Buffalari and Grace, 2007) and potentially modulates the VTA via the nucleus accumbens (NAc) (Sesack and Grace, 2010) and other structures (Condé et al., 1995; Mcdonald et al., 1996; Pitkänen et al., 2000; Herry et al., 2008). The BLA is well known for its involvement in aversive (stressful) learning and is highly modulated by norepinephrine (NE) (McGaugh, 2004; Roozendaal et al., 2006; Tully and Bolshakov, 2010; Dębiec et al., 2011; Johnson et al., 2011), placing it as a strong candidate in mediating the interaction between the noradrenergic and dopaminergic systems.

Using the combined techniques of electrophysiological recordings, behavioral testing, pharmacological manipulations, and immunohistochemical approaches, we examined the delayed response of the VTA DA neurons to $2 \mathrm{~h}$ acute restraint and the modulatory role of the noradrenergic system in this response. We hypothesize that the immediate increase of the VTA DA neuron population activity and amphetamine-induced locomotor activity transition over time to a different, opposite homeostatic state that may be related to NE actions within the BLA.

\section{Materials and Methods}

Subjects and materials. A total of 212 male Sprague Dawley rats (300-400 g; Harlan Laboratories) were used in this study: 72 rats were used for electrophysiological recordings, 128 rats for behavioral testing, and 12 rats for immunohistochemistry. Rats were housed for at least $5 \mathrm{~d}$ in pairs in a temperature-controlled $\left(22^{\circ} \mathrm{C}\right)$ and humidity-controlled $(47 \%) \mathrm{fa}-$ cility upon arrival, with lights maintained on a $12 \mathrm{~h}$ light/dark cycle (lights on at 7:00 A.M.) and food and water available ad libitum. Han- 
dling of all animals, surgery, and experiment protocols were in accordance with the guidelines outlined in the United States Public Health Service Guide for the Care and Use of Laboratory Animals, and were approved by the Institutional Animal Care and Use Committee of the University of Pittsburgh.

Stress paradigms. A psychological stressor of acute restraint was used on awake rats. Rats were restrained in Plexiglas cylinders (internal diameter of $6.2 \mathrm{~cm}$, length adjusted accordingly to rat size; IITC Life Science) for a $2 \mathrm{~h}$ session between 9:00 A.M. and 12:00 P.M., across all studies. Control animals were placed in the holding cage for an equivalent $2 \mathrm{~h}$ period. Our protocol states that any rats showing excessive distress in terms of extended vocalizations or struggling should be removed from the study; however, none of the rats fit the criteria for exclusion. For immunohistochemical studies, rats were restrained for a 30 min session, which has been shown effective at inducing $\mathrm{c}$-fos expression in regions of interests (Goebel et al., 2009).

Acute surgery and electrophysiological recordings from anesthetized rats. Single-unit extracellular recordings were performed on anesthetized rats between 9:00 A.M. and 5:00 P.M. (Valenti et al., 2011). Rats were anesthetized with $8 \%$ chloral hydrate $(400 \mathrm{mg} / \mathrm{kg}$, i.p.) and placed in a stereotaxic apparatus (David Kopf Instruments); core body temperature of $37^{\circ} \mathrm{C}$ was maintained by a temperature-controlled heating pad (FST). Supplemental injections were administered as needed. The skull overlying the recording region in the VTA [from bregma: anteroposterior (AP), $-5.3 \mathrm{~mm}$; mediolateral (ML), $-0.6 \mathrm{~mm}$ ] was removed, and the dura was resected. Single-barrel electrodes ( $2 \mathrm{~mm}$ outer diameter Omegadot filament glass; World Precision Instruments) were constructed using a vertical microelectrode puller (PE-2; Narishige), and the recording barrel filled with $2 \%$ Pontamine sky blue in $2 \mathrm{M} \mathrm{NaCl}$ with in situ impedance of 4-10 $\mathrm{M} \Omega$ (measured at $1 \mathrm{kHz}$ ). Glass electrodes were lowered into the VTA [from brain surface: dorsoventral (DV), -6.5 to $-9.0 \mathrm{~mm}$ ] using a hydraulic microdrive (David Kopf Instruments, model 640). Signals from the recording electrode were amplified by a headstage before being fed into a window discriminator/amplifier (1000 gain, $50-16 \mathrm{kHz}$ bandpass; Fintronics), then into an audio monitor (Grass Instruments, model AM8), and displayed on an oscilloscope (Tektronix) for realtime monitoring. Data were collected using a data acquisition board interface, monitored on-line, and analyzed off-line using computer software (PowerLab; AD Instruments).

DA neurons were identified with open filter setting (highpass, $50 \mathrm{~Hz}$; lowpass, 16k Hz) and distinguished from other VTA neurons based on their unique long-duration waveform, slow irregular firing rate, and other well established criteria (Grace and Bunney, 1983; Grace et al., 2007; Ungless and Grace, 2012). Three parameters of VTA DA neuron activity were calculated. (1) Population activity was assessed by passing the electrode through the VTA in a predetermined grid pattern of six to nine tracks separated by $200 \mu \mathrm{m}$; all spontaneously active DA neurons encountered per electrode track were counted. After isolating a DA neuron, $3 \mathrm{~min}$ of activity was recorded to determine the (2) firing rate and (3) percentage of burst firing. Percentage of burst firing was quantified by examining the proportion of action potentials occurring in bursts, with bursts defined as the occurrence of two spikes with an interspike interval (ISI) of $<80 \mathrm{~ms}$ indicating the initiation of a burst, and two spikes with an ISI $>160$ ms signaling burst termination (Grace and Bunney, 1984).

Locomotor response to psychostimulants. The locomotor response to psychostimulants was assessed as described previously (Lodge and Grace, 2008). Rats were housed with a reverse $12 \mathrm{~h}$ light/dark cycle (lights on 7:00 P.M.) for at least 1 week before behavioral experiments. On testing days (9:00 A.M. to 5:00 P.M.), rats were acclimatized to an open-field arena (Coulbourn Instruments) where spontaneous locomotor activity in the $X-Y$ plane was monitored for $30 \mathrm{~min}$ by beam breaks and recorded with TruScan software (Coulbourn Instruments). Rats were then administered either a challenge dose of amphetamine $(0.5 \mathrm{mg} / \mathrm{kg}$, i.p.) or saline (control animals, i.p.), and locomotor activity was recorded for an additional 90 min (Lodge and Grace, 2007). The order of testing was counterbalanced across groups. It should be noted that locomotor activity was examined during the active part of the diurnal cycle, whereas DA recordings were performed during the inactive part of the cycle. Importantly, recent data have demonstrated that DA neurons do not display differences in firing across the diurnal cycle (Luo et al., 2008).

Survival surgery and drug administration. The $\beta$-noradrenergic receptor antagonist, propranolol (Sigma), was administered either systemically or intra-BLA. For systemic administration, drugs were dissolved in sterile saline to a final concentration of $5 \mathrm{mg} / \mathrm{ml}$. Rats were given propranolol ( $5 \mathrm{mg} / \mathrm{kg}$, i.p.) or saline as the control. For intra-BLA administration, rats were implanted bilaterally with 26 gauge stainless steel guide cannulae (Plastics One) targeting the BLA (from the bregma: AP, -2.9 $\mathrm{mm}$; ML, $+5.0 \mathrm{~mm}$; DV,$-7.6 \mathrm{~mm}$ ) under isoflurane inhalation anesthesia (Halocarbon Laboratories; $1-3 \%$ in oxygen). Four additional anchor screws (FST) were mounted and the headstage was fixed in place with dental cement. Rats were then administered postoperative analgesic ( $2 \mathrm{mg} / \mathrm{kg}$ ketoprofen, s.c. and 10\% Tylenol Children's Syrup added to rat chow). At least $7 \mathrm{~d}$ of recovery were allowed before drug administration. Injectors (33 gauge) protruding $1.0 \mathrm{~mm}$ past the end of the implanted guide cannulae were inserted and the rats received infusions of propranolol ( $0.5 \mu \mathrm{g}$ per side) or saline as the control. All drugs were delivered in $0.2 \mu \mathrm{l}$ of sterile saline $(0.1 \mu \mathrm{l} / \mathrm{min})$, and another minute was allowed for diffusion of the drug before the internal injectors were removed. The dosages used have been proven effective in earlier studies (Salmon and Stanford, 1989; Roozendaal et al., 2006).

Histology. At the cessation of the electrophysiology experiments, the recording site was marked via electrophoretic ejection (Kation Scientific, model BAB-501) of Pontamine sky blue dye from the tip of the recording electrode ( $-20 \mu \mathrm{A}$ constant current for $40 \mathrm{~min}$ ). Rats were killed by an overdose of anesthetic (chloral hydrate, additional $400 \mathrm{mg} / \mathrm{kg}$, i.p.) or $\mathrm{CO}_{2}$. All rats were decapitated, and their brains were removed, fixed for at least $48 \mathrm{~h}$ [ $8 \%$ paraformaldehyde (PFA) in PBS], and cryoprotected (25\% sucrose in PBS) until saturated. Brains were sectioned $(60 \mu \mathrm{m}$ coronal sections), mounted onto gelatin-chrome alum-coated slides and stained with cresyl violet for histochemical verification of electrode and/or cannula sites.

Immunohistochemistry. Rats were anesthetized by isoflurane inhalation (Halocarbon Laboratories; $1-3 \%$ in oxygen) and oriented in a Kopf stereotaxic instrument in the flat skull position. A small hole $(\sim 1-2 \mathrm{~mm}$ diameter) was opened in the skull to expose the cortical surface overlying the BLA (from bregma: AP, $-2.9 \mathrm{~mm}$; ML, $-5.0 \mathrm{~mm}$; DV, $-8.6 \mathrm{~mm}$ ) tracer delivery target site. A pulled glass micropipette $(\sim 20 \mu \mathrm{m}$ outer tip diameter) was attached to the arm of the stereotaxic apparatus. Using negative pressure, the micropipette was backfilled with a $1 \%$ solution of the retrograde tracer of fluorogold (FG; Fluorochrome) diluted in $0.1 \mathrm{M}$ cacodylic acid, and then a microwire connected to a current source (Stoelting) was lowered into the tracer solution. FG was unilaterally delivered by iontophoresis into the BLA using a $7 \mathrm{~s}$ pulsed positive current of $5 \mu \mathrm{A}$ for a duration of $5 \mathrm{~min}$. Iontophoretic parameters were determined through pilot studies and were found to produce discrete, localized tracer delivery sites with minimal tracer diffusion into adjacent regions. The micropipette tip was left in place for $1 \mathrm{~min}$ after iontophoresis, and then was withdrawn. The skin over the skull was closed with stainless steel clips and rats were injected postoperatively with analgesic ( $2 \mathrm{mg} / \mathrm{kg}$ ketoprofen, s.c.). Rats were returned to their home cage after regaining consciousness and full mobility.

After a 10-14 d postiontophoresis survival time, the rats were subjected to the restraint procedures. To capture the time window of maximal c-fos expression 90 min after the manipulation (Knapska and Maren, 2009), the restraint protocol was modified with half of the rats restrained in Plexiglas cylinders for a $30 \mathrm{~min}$ session, returned to their home cage, and left undisturbed for $1 \mathrm{~h}$, while the other half remained in their home cage throughout the time interval. All rats were then deeply anesthetized with sodium pentobarbital (Nembutal, $100 \mathrm{mg} / \mathrm{kg}$, i.p.) and perfused transcardially with $100 \mathrm{ml}$ of saline followed by $500 \mathrm{ml}$ of fixative $(4 \%$ PFA in $0.1 \mathrm{M}$ PBS). Brains were postfixed overnight at $4^{\circ} \mathrm{C}$ and cryoprotected in $20 \%$ sucrose solution for $24-72 \mathrm{~h}$. Brains were sectioned coronally $(35 \mu \mathrm{m})$ using a freezing microtome. Sections were collected sequentially into six adjacent sets and were stored in cryopreservant at $-20^{\circ} \mathrm{C}$ for later immunohistochemical processing.

One tissue section series (containing sections spaced by $210 \mu \mathrm{m}$ ) from each rat was used for dual immunoperoxidase localization of nuclear 

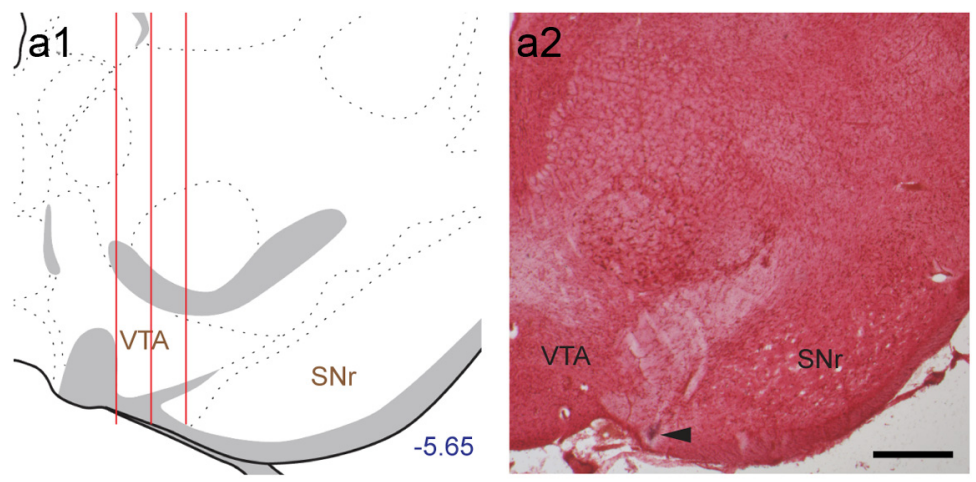

b1

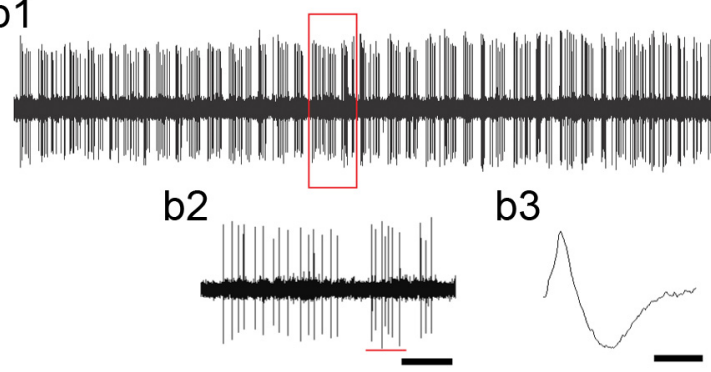

Figure 1. Evaluation of VTA DA neuron activity states. $\boldsymbol{a} 1, \mathrm{DA}$ neurons were sampled with predetermined grid pattern of six $(3 \times 2)$ to nine $(3 \times 3)$ tracks separated by $200 \mu \mathrm{m}$. $\boldsymbol{a 2}$, A representative Pontamine sky blue deposit. Arrowhead indicates the final location of the electrode. Scale bar, $500 \mu \mathrm{m} . \boldsymbol{b} 1$, A segment of spontaneous VTA DA activity. Scale bar, $10 \mathrm{~s}$. $\boldsymbol{b 2}$, A closer view of DA activity. Underlying red line indicates a burst event. Calibration: $1 \mathrm{~s} . \mathbf{b 3}$, A representative waveform of a DA neuron. Scale bar, $1 \mathrm{~ms}$. SNr, Substantia nigra pars compacta.

c-fos protein and cytoplasmic neural tracer, FG. Tissue sections were removed from storage and rinsed in buffer $(0.1 \mathrm{~m}$ sodium phosphate, $\mathrm{pH}$, 7.4) for $1 \mathrm{~h}$ before immunohistochemical procedures. Immunoperoxidase localization of c-fos protein followed previously established protocols (Bienkowski and Rinaman, 2011). Tissue sections were incubated overnight in buffer containing $0.3 \%$ Triton X-100, $1 \%$ normal donkey serum, $1 \%$ bovine serum albumin, and a rabbit polyclonal anti-c-fos antibody (1:50,000; Dr. Philip Larsen, Denmark). After rinsing with buffer, sections were incubated in biotinylated donkey anti-rabbit IgG (1: 500; Jackson ImmunoResearch) and Vectastatin ABC Elite reagents (Vector Laboratories) followed by a solution of nickel sulfate and diaminobenzidine (DAB) with hydrogen peroxide to produce a blue-black reaction product within the nucleus of c-fos-positive neurons. Sections were then rinsed in buffer and incubated overnight in buffer containing $0.3 \%$ Triton $\mathrm{X}-100,1 \%$ normal donkey serum, and rabbit anti-FG (1: 30,000; Millipore Bioscience Research Reagents) to localize neural tracer. After rinsing, sections were incubated in biotinylated secondary donkey anti-rabbit IgG (1:500) and Vectastatin ABC Elite reagents followed by a nonintensified $\mathrm{DAB}$ - hydrogen peroxide reaction to produce brown immunoprecipitate localizing the neural tracer delivery site and retrogradely labeled neurons. Immunostained tissue sections were rinsed in buffer, mounted onto Superfrost Plus microscope slides (Fisher Scientific), allowed to dry overnight, dehydrated and defatted in graded ethanols and xylene, and coverslipped using Cytoseal 60 (VWR).

Dual-immunoperoxidase-labeled tissue sections from each rat were analyzed using a light microscope to determine the number and proportion of retrogradely labeled BLA afferents activated to express c-fos after restraint. Criteria for counting tracer-positive neurons included the presence of brown cytoplasmic immunoreactivity and a visible nucleus. Tracer-positive neurons in which the nucleus contained blue-black c-fos immunoreactivity were considered double labeled, regardless of c-fos labeling intensity. Tracer-positive neurons were quantified in sections spaced by $210 \mu \mathrm{m}$ through the LC (from bregma: AP, $\sim-9.5 \mathrm{~mm}$ to $-10.1 \mathrm{~mm}$ ), comprising $2-3$ tissue sections (spaced by $210 \mu \mathrm{m}$ ) per rat.

Data analysis and statistics. Electrophysiological analysis of single-unit neuron activities was performed using computer software, PowerLab (AD instruments) and Nex (NEX Technologies). Locomotor behavior was recorded using TruScan software (Coulbourn Instruments). For immunohistochemical data, the total number of single-labeled (tracer only) and double-labeled (tracer + c-fos) neurons in LC was counted. The percentage of activated neurons was calculated as $($ tracer $+\mathrm{c}-f o s) /[($ tracer only $)+($ tracer + c-fos) $] \times 100$. All data are represented as the mean \pm SEM unless otherwise stated and were submitted to ANOVA or $t$ test. Post hoc comparisons in the form of Neuman-Keuls tests were performed after a significance of $p<0.05$. All statistics were calculated using SPSS (IBM).

\section{Results}

\section{VTA DA neurons}

VTA DA activity was assessed by passing the electrode through the VTA in a predetermined grid pattern of six $(3 \times 2)$ to nine $(3 \times 3)$ tracks separated by $200 \mu \mathrm{m}$ (Valenti et al., 2011; Fig. 1a1,a2). Population activity (number of spontaneously firing DA neurons per electrode track), mean firing rate, and percentage of burst firing (i.e., the proportion of action potentials occurring in bursts, with burst onset defined as the occurrence of two spikes with an ISI of $<80 \mathrm{~ms}$, and termination defined as two spikes with an ISI $>160$ ms; Grace and Bunney, 1984) were calculated (Fig. 1b1,b2). DA neurons (Fig. 1b3) were identified with open filter setting (highpass, $50 \mathrm{~Hz}$; lowpass, $16 \mathrm{k} \mathrm{Hz}$ ) and distinguished from other VTA neurons based on their unique long-duration waveform $(>2.5 \mathrm{~ms})$, slow irregular firing rate $(<5 \mathrm{~Hz})$, and other well established criteria (Grace and Bunney, 1983; Grace et al., 2007). By using this standard set of criteria, we were able to accurately identify the large majority of DA neurons reported in this study (Ungless and Grace, 2012).

\section{A significant decrease in VTA DA neuron population activity and attenuated amphetamine-induced locomotor activity is present $24 \mathrm{~h}$ after acute restraint stress}

Previously, we found that $2 \mathrm{~h}$ of acute restraint significantly increased VTA DA neuron population activity and amphetamineinduced locomotor activity when tested immediately after the stressful event (Valenti et al., 2011). In this study, we further explored whether this enhancement is transient or long lasting by testing these responses $24 \mathrm{~h}$ after the acute restraint.

The subjects were 58 male Sprague Dawley rats: 18 rats were used for DA electrophysiological recordings and 40 rats for behavioral testing. For electrophysiological recordings, rats were assigned into one of the two groups: one that was restrained for a $2 \mathrm{~h}$ session (Res, $n=10$ ), and a control group that was held in the holding cage for the equivalent amount of time without restraint (NoRes, $n=8$ ).

In contrast to the increased DA neuron population activity observed soon after restraint, we found a significant decrease in population activity $24 \mathrm{~h}$ after the $2 \mathrm{~h}$ acute restraint by $\sim 50 \%$ (Fig. $2 a 1 ; t_{(16)}=-2.43, p=0.03$ ). A total of 53 DA neurons were recorded in the Res group and 83 DA neurons in the NoRes group. No differences in the average firing rate (Fig. $2 a 2 ; t_{(134)}=$ $0.22, p=0.83$ ) or percentage of spikes in bursts (Fig. $2 a 3 ; t_{(134)}=$ $0.51, p=0.61)$ was observed.

Multiple studies have shown that the level of DA neuron population activity correlates with the behavioral responses to 
amphetamine (Lodge and Grace, 2007, 2008; Valenti et al., 2011; Cifelli and Grace, 2012). To provide additional data to support the finding of a delayed decrease in DA system activity, we examined whether such a decrease corresponded to a decreased behavioral response to amphetamine.

The same procedure was used for Res and NoRes for the locomotor behavioral testing. One NoRes-saline (SAL) rat was lost due to computer error, yielding the final group sizes: Res-SAL $(n=10)$, Resamphetamine (AMPH) $(n=10)$, NoResSAL $(n=9)$, and NoRes-AMPH $(n=10)$.

We found that following administration of $0.5 \mathrm{mg} / \mathrm{kg}$ amphetamine, only rats in the NoRes group displayed significantly higher levels of locomotor activity compared with their saline controls (Fig. 2b). For the 30 min acclimation, there was an equivalent decrease in locomotor activity across sampling blocks ( $5 \mathrm{~min}$ bin) in all groups (Restraint $\times$ Drug $\times$ Sampling Block, $\left.F_{(5,175)}<1\right)$. For the 90 min postinjection testing, an ANOVA revealed a significant main effect of Drug $\left(F_{(1,35)}=6.62, p=0.01\right)$, and a significant three-way interaction among Restraint, Drug, and Sampling Block $\left(F_{(17,595)}=1.67, p=0.045\right)$. Planned comparisons suggested that there was no significant difference between saline controls (Res-SAL and NoRes-SAL, $p=0.55$ ). The locomotor activity was only significantly higher after administering amphetamine in NoRes rats compared with their saline controls (Fig. 2b1; NoRes-AMPH and NoRes-SAL, $p=0.006$ ), but not in Res rats (Fig. 2b2; Res-AMPH and Res-SAL, $p=0.51$ ). The effect was most robust at the onset of amphetamine administration, which wore off at later time points. Planned comparisons demonstrated significantly higher locomotor activity in NoRes-AMPH rats compared with Res-AMPH rats between postinjection sampling blocks of $10-20 \mathrm{~min}$ ( $p$ s $<0.05$ ).

\section{Prerestraint systemic administration of the $\boldsymbol{\beta}$-noradrenergic receptor antagonist, propranolol, blocked the decrease in VTA DA neuron population activity and prevented the attenuation of amphetamine-induced locomotor activity observed $24 \mathrm{~h}$ after restraint}

Given that stressful events are known to lead to increased release of noradrenaline (Morilak et al., 2005; Roozendaal et al., 2009), we tested whether prerestraint blockade of noradrenaline with systemic administration of the $\beta$-noradrenergic receptor antagonist, propranolol, prevented the decrease in VTA DA neuron population activity $24 \mathrm{~h}$ after acute restraint.

The subjects were 41 male Sprague Dawley rats: 17 rats were used for DA electrophysiological recordings and 24 rats for behavioral testing. For electrophysiological recordings, all rats were restrained for a $2 \mathrm{~h}$ session. Half of the rats were administered propranolol systemically prerestraint ( $\mathrm{PRO}, n=8 ; 5 \mathrm{mg} / \mathrm{kg}$, i.p.), and the other half with saline as the control (SAL, $n=9$; i.p.).

We found that prerestraint blockade of the noradrenaline $\beta$-receptors blocked the delayed decrease in VTA DA neuron population activity, which was significantly higher by $130 \%$ in animals pretreated with propranolol compared with their saline controls (Fig. 3a1). There was a significant difference between
SAL and PRO $\left(t_{(15)}=-3.15, p=0.007\right)$. Indeed, in PRO-treated rats, DA neuron population activity was nearly identical to that of NoRes control rats in the first experiment (95\% confidence interval from 0.87 to 1.48 ). A total of $42 \mathrm{DA}$ neurons were recorded in the SAL group and 78 DA neurons in the PRO group. Propranolol administration did not affect the average firing rate (Fig. 3a2; $t_{(118)}=1.17, p=0.24$ ) or percentage of burst firing (Fig. 3a3; $\left.t_{(118)}=1.11, p=0.27\right)$. In contrast, in naive rats that did not receive the restraint stress, there was no difference between propranolol- and saline-treated animals with respect to DA neuron population activity [SAL, $1.02 \pm 0.12(n=6) ; \mathrm{PRO}, 1.04 \pm$ $\left.0.30(n=6) ; t_{(10)}=0.06, p=0.96\right]$, average firing rate [SAL, $4.25 \pm 0.34(n=55) ;$ PRO, $4.72 \pm 0.29(n=56) ; t_{(109)}=1.07$, $p=0.29$ ], or percentage of bursting firing [SAL, $31.67 \pm 3.99$ $(n=55)$; PRO, $\left.31.45 \pm 3.93(n=56) ; t_{(109)}=0.04, p=0.97\right]$. Therefore, the effect of propranolol was not due to nonspecific action of noradrenergic signaling independent of the restraint stress.

Given that prerestraint systemic propranolol administration blocked the decrease in DA neuron population activity $24 \mathrm{~h}$ after restraint, we examined whether this treatment also prevented the decreased behavioral response to amphetamine. All rats were restrained for a $2 \mathrm{~h}$ session, and the same procedure was used for PRO and SAL for the locomotor behavioral testing. One PROSAL rat was lost to computer error, and one SAL-AMPH rat failed to acclimate to the open arena during the first $30 \mathrm{~min}$, yielding the final group sizes: SAL-SAL $(n=6)$, SAL-AMPH $(n=5)$, PROSAL $(n=5)$, and PRO-AMPH $(n=6)$.

We found that propranolol pretreated rats showed a more robust increase in amphetamine-induced locomotor activity compared with saline pretreated controls (Fig. $3 b$ ) when tested $24 \mathrm{~h}$ after the cessation of the acute restraint. For the $30 \mathrm{~min}$ acclimation, there was an equivalent decrease in locomotor activity across sampling blocks ( $5 \mathrm{~min}$ bin) in all groups (Treatment $\times$ Drug $\times$ Sampling Block, $\left.F_{(5,90)}<1\right)$. For the $90 \mathrm{~min}$ postinjection testing, an ANOVA revealed a significant main effect of Treatment $\left(F_{(1,18)}=6.12, p=0.024\right)$, a significant main 
a1

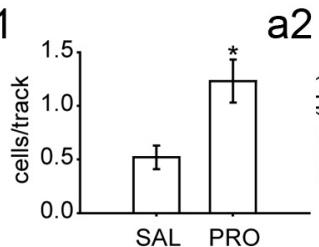

b1

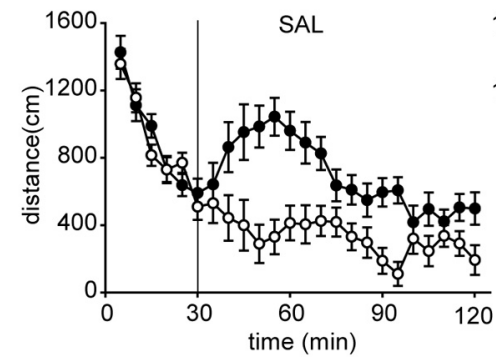

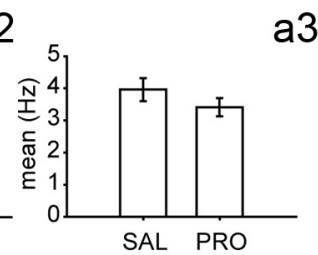

b2 a3

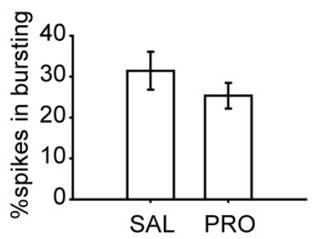

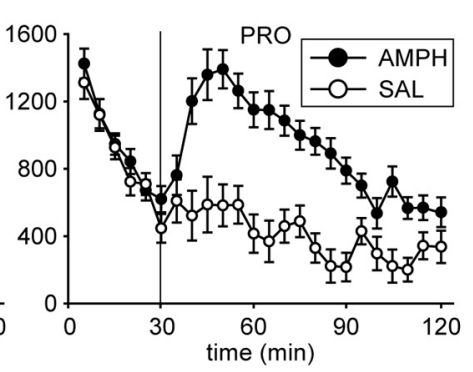

Figure 3. Prerestraint systemic administration of the $\beta$-noradrenergic receptor antagonist, propranolol, blocked the decrease in VTA DA neuron population activity and prevented the attenuation of amphetamine-induced locomotor activity measured $24 \mathrm{~h}$ after restraint. $\boldsymbol{a 1 - a 3}$, For all rats restrained for a $2 \mathrm{~h}$ session, propranolol pretreatment (PRO; $n=8$ ) significantly increased the DA neuron population activity compared with saline $(S A L ; n=9)$ control $\left({ }^{*} p<0.05\right)(\boldsymbol{a} 1)$, with no significant difference between mean firing rates ( $\boldsymbol{a} 2$ ) or bursting activity (a3). DA neurons recorded: SAL $(n=42)$, PRO $(n=78) . \boldsymbol{b} 1, \boldsymbol{b} 2$, AMPH induced higher locomotor activity regardless pretreatment (b1, SAL; $\boldsymbol{b 2}$, PRO), with significantly higher locomotor activity produced in rats pretreated with PRO. (all ps < 0.05). Group sizes: SAL-SAL $(n=6), \operatorname{SAL-AMPH~}(n=5), \operatorname{PRO-SAL}(n=5)$, and PRO-AMPH $(n=6)$.

effect of Drug $\left(F_{(1,18)}=50.04, p<0.001\right)$, and a significant twoway interaction between Drug and Sampling Block $\left(F_{(17,306)}=\right.$ 5.04, $p<0.001)$. Planned comparisons suggested that there was no significant difference between saline controls regardless of prerestraint treatment (SAL-SAL and PRO-SAL, $p=0.41$ ). Unlike in the first experiment in which $2 \mathrm{~h}$ acute restraint completely blocked the response to amphetamine (Fig. 2b2), in this study amphetamine induced an increased locomotor activity compared with their saline controls regardless of prerestraint treatment (Fig. 3b1, SAL-SAL and SAL-AMPH, $p=0.001$; Fig. 3b2, PRO-SAL and PRO-AMPH, $p<0.001)$. However, following amphetamine administration, there was a significantly higher locomotor activity in propranolol pretreated rats compared with saline pretreated rats (increase by $34 \%$; Fig. 3b1,b2, PRO-AMPH and SAL-AMPH, $p=0.016)$.

Postrestraint systemic administration of the $\boldsymbol{\beta}$-noradrenergic receptor antagonist, propranolol, blocked the decrease in VTA DA neuron population activity and prevented the attenuation of amphetamine-induced locomotor activity $24 \mathrm{~h}$ following restraint

Stress-induced monoaminergic release generally occurs within minutes after the onset of the stressor and seldom outlasts the duration of stressor exposure (Joëls and Baram, 2009). In the previous experiment, prerestraint blockade of $\beta$-noradrenergic receptors prevented the decrease in VTA DA neuron population activity and the attenuated response to amphetamine $24 \mathrm{~h}$ after restraint. However, to be an effective treatment of a stressinduced depression, a manipulation should be effective when administered after the stressor. Thus, we examined whether blockade of noradrenaline immediately after the release from a $2 \mathrm{~h}$ acute restraint session is sufficient and effective in blocking the attenuation of the DA system observed $24 \mathrm{~h}$ after restraint.

The subjects were 50 male Sprague Dawley rats: 18 rats were used for DA electrophysiological recordings and 32 rats for behavioral testing. For electrophysiological recordings, all rats were restrained for a $2 \mathrm{~h}$ session. Eight of the rats were administered

propranolol systemically postrestraint (PRO, $n=8 ; 5 \mathrm{mg} / \mathrm{kg}$, i.p.), and the others with saline as the control (SAL, $n=10$; i.p.).

Similar to that observed with prerestraint blockade of noradrenaline, the population activity in propranolol posttreated rats was increased by $60 \%$ and restored to values that are within a 95\% confidence interval (CI) of NoRes control rats in the first study (Fig. 4a1). A total of 52 DA neurons were recorded in the SAL group and $56 \mathrm{DA}$ neurons in the PRO group. Propranolol administration did not affect the average firing rate (Fig. 4a2; $t_{(106)}=1.26, p=0.21$ ) but significantly decreased the percentage of spikes in bursts (Fig. $4 a 3 ; t_{(106)}=2.64, p=0.01$ ).

All rats were restrained for a $2 \mathrm{~h}$ session, and the same procedure was used for PRO and SAL for the locomotor behavioral testing. Injection error occurred in one of the propranolol-treated rats leading to unknown dosage injected and thus this rat was excluded from data analysis, yielding the final group sizes: SAL-SAL $(n=8)$, SAL-AMPH $(n=8)$, PRO-SAL $(n=8)$, and PRO$\operatorname{AMPH}(n=7)$.

We found that rats treated with propranolol after restraint showed a more robust increase in amphetamine-induced locomotor activity compared with saline post-treated controls $24 \mathrm{~h}$ after the cessation of the acute restraint (Fig. 4b). For the $30 \mathrm{~min}$ acclimation, there was an equivalent decrease in locomotor activity across sampling blocks ( $5 \mathrm{~min}$ bin) in all groups (Treatment $\times$ Drug $\times$ Sampling Block, $\left.F_{(5,135)}<1\right)$. For the $90 \mathrm{~min}$ after injection testing, an ANOVA revealed a significant main effect of Drug $\left(F_{(1,27)}=41.62, p<0.001\right)$, and a significant twoway interaction between Drug and Sampling Block $\left(F_{(17,459)}=\right.$ $6.62, p<0.001)$. Planned comparisons suggested that there was no significant difference between saline controls regardless of postrestraint treatment (SAL-SAL and PRO-SAL, $p=0.67$ ). Similar to the results obtained with propranolol pretreatment, in this study amphetamine induced an increased locomotor activity compared with their saline controls regardless of postrestraint treatment (Fig. 4b1, SAL-SAL and SAL-AMPH, $p=0.003$; Fig. $4 b 2$, PRO-SAL and PRO-AMPH, $p<0.001)$. However, following amphetamine administration, there was a significantly higher locomotor activity in propranolol post-treated rats compared with saline post-treated rats (increase by $32 \%$; Fig. 4b1,b2, PROAMPH and SAL-AMPH, $p=0.031)$.

\section{Acute restraint activated LC neurons projecting to the BLA}

The previous two experiments demonstrated that systemic propranolol administration prerestraint and postrestraint effectively blocked the stress-induced decrease in DA system activity $24 \mathrm{~h}$ following restraint. Given that the noradrenergic neurons in the LC are activated by stressful events (Rasmussen et al., 1986; Aston-Jones et al., 1991; Morilak et al., 2005; Goebel et al., 2009), we first established whether LC neurons that project directly from the LC to the BLA are activated by acute restraint.

The subjects were 12 male Sprague Dawley rats. FG retrograde tracer was injected into the BLA (targeting the basal nucleus) and half of the rats were subjected to a $30 \mathrm{~min}$ restraint session. c-fos 
activation was examined $30 \mathrm{~min}$ after restraint, although $2 \mathrm{~h}$ of restraint were used in the electrophysiological study. This was necessary to examine maximal c-fos activation, which is transient in nature ( $\max -$ imal at $90 \mathrm{~min}$ ), but should nonetheless reveal which systems are activated during this exposure. Three rats were excluded because the tracer missed the target of the BLA, yielding the final group sizes: Res $(n=5)$ and NoRes $(n=4)$.

A representative tracer delivery site is shown in Figure 5a1, and an illustration of the largest and smallest sizes of tracer spread is shown in Figure 5a2. Tracers were discrete and localized to the delivery sites with minimal diffusion into adjacent regions. Retrogradely labeled BLA afferents were found in the LC (Fig. 5b1,b2). The number of retrogradely labeled LC neurons was equivalent in both Res and NoRes groups (Fig. 5c1). There was no significant difference between Res and NoRes in tracer-positive neurons [(tracer only $)+($ tracer $+c-f o s) ; t_{(7)}=-0.74, p=$ 0.483]. However, among the tracerpositive neurons in the $\mathrm{LC}$, more neurons were activated by acute restraint as indicated by positive c-fos immunoreactivity (Fig. $5 c 2, c 3)$. There was a significant difference between dual-labeled neurons (tracer $+c$-fos) between Res and NoRes (Figure $5 c 2$; $\left.t_{(7)}=4.23, p=0.004\right)$. Acute restraint activated a significantly higher percentage of the LC neurons that project directly onto the BLA (Figure $5 c 3 ; t_{(7)}=7.49, p<0.001$ ).

Prerestraint intra-BLA administration of the $\boldsymbol{\beta}$-noradrenergic receptor antagonist, propranolol, blocked the decrease in VTA DA neuron population activity and prevented the attenuation of amphetamine-induced locomotor activity $24 \mathrm{~h}$ following restraint

From the experiments above, we showed that systemic administration of propranolol prevented the attenuation of DA system activity $24 \mathrm{~h}$ after restraint. Moreover, the direct projection from the LC onto the BLA is highly activated after the acute restraint session, placing the BLA as a strong candidate for mediating the LC NE effects on VTA DA neuron activity. In this study, we examined whether prerestraint blockade of $\beta$-noradrenergic receptors in the BLA is sufficient and effective at blocking the decrease in VTA DA neuron population activity and preventing the attenuation of amphetamine-induced locomotor activity $24 \mathrm{~h}$ following restraint stress.

The subjects were 51 male Sprague Dawley rats bilaterally implanted with guiding cannulae targeting the BLA: 19 rats were used for DA electrophysiological recordings and 32 rats for behavioral testing. For electrophysiological recordings, all rats were restrained for a $2 \mathrm{~h}$ session. Half of the rats were administered intra-BLA propranolol prerestraint (PRO, $n=9 ; 0.5 \mu \mathrm{g} / 0.2 \mu \mathrm{l}$ per side), and the other half were administered with saline as the control (SAL, $n=10 ; 0.2 \mu$ l per side).

The placements of injection sites from all rats included in this study are shown in Figure 6a1. Given the small volume of injection compared with other relevant studies (Roozendaal et al., 2008; Zimmerman and Maren, 2010), the spread of the infused drug is estimated to remain well within the confines of the BLA. We found that, as with systemic administration, DA neuron pop- ulation activity was significantly higher in animals pretreated with propranolol compared with their saline controls by $62 \%$ and restored to values that are within a $95 \% \mathrm{CI}$ of NoRes control rats in the first study (Fig. 6a2). There was a significant difference between SAL and PRO $\left(t_{(17)}=-2.65, p=0.017\right)$. A total of 59 DA neurons were recorded in SAL group and 86 DA neurons in PRO group. Propranolol administration did not affect the average firing rate (Fig. $6 a 3 ; t_{(143)}=0.22, p=0.823$ ) or percentage of spikes in bursts (Fig. $6 a 4 ; t_{(143)}=-0.96, p=0.337$ ).

We next examined whether the intra-BLA propranolol administration prerestraint also normalized behavioral response to amphetamine. All rats were restrained for a $2 \mathrm{~h}$ session, and the same procedure was used for PRO and SAL for the locomotor behavioral testing. The cap of one rat in the SAL-SAL group fell off and was thus excluded from data analysis, yielding the final group sizes: SAL-SAL $(n=7)$, SAL-AMPH $(n=8)$, PRO-SAL $(n=8)$, and PRO-AMPH $(n=8)$.

The placements of injection sites from all rats included in this study are shown in Figure 6b1. We found that, compared with saline pretreated controls, propranolol pretreated rats showed a more robust increase in amphetamine-induced locomotor activity $24 \mathrm{~h}$ after the cessation of the acute restraint (Fig. 6b). For the 30 min acclimation, there was an equivalent decrease in locomotor activity across sampling blocks (5 min bin) in all groups (Treatment $\times$ Drug $\times$ Sampling Block, $F_{(5,135)}<1$ ). For the 90 min postinjection testing, an ANOVA revealed a significant main effect of Drug $\left(F_{(1,27)}=26.8, p<0.001\right)$, a significant two-way interaction between Treatment and Drug $\left[F_{(1,27)}=4.62, p=\right.$ $0.041]$, and a significant two-way interaction between Drug and Sampling Block $\left(F_{(17,459)}=4.28, p<0.001\right)$. Planned comparisons suggested that there was no significant difference between saline controls regardless of prerestraint treatment (SAL-SAL and PRO-SAL, $p=0.699$ ). Similar to the results of the earlier systemic propranolol treatment experiments, in this study amphetamine induced an increase in locomotor activity compared with their saline controls regardless of intra-BLA pretreatment (Fig. 6b2, SAL-SAL and SAL-AMPH, $p=0.045$; Fig. 6b3, PRO-SAL and 

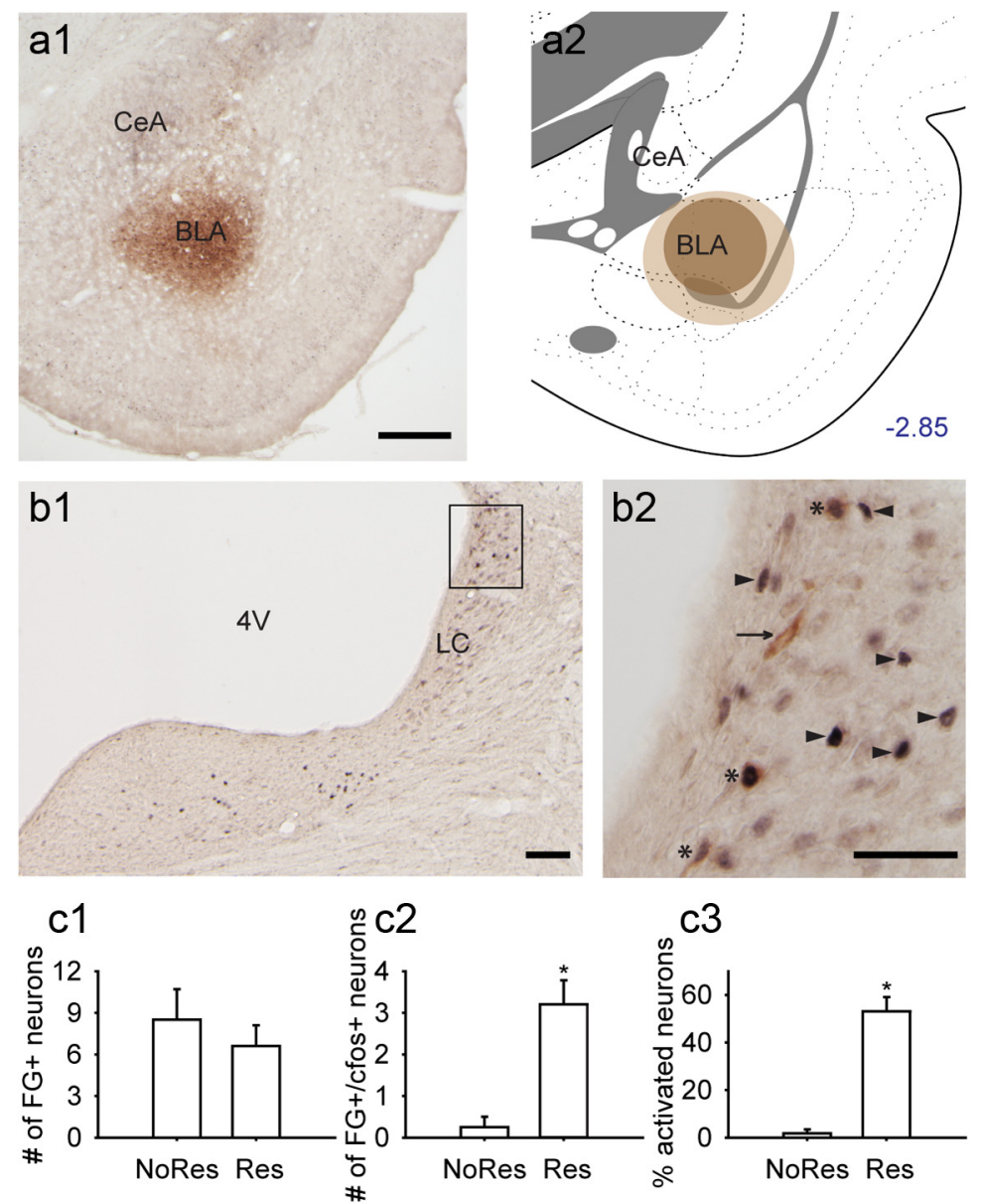

Figure 5. Acute restraint activated LC neurons projecting to the BLA. a1, A representative tracer delivery site of FG into the BLA. Scale bar, $500 \mu \mathrm{m} . \boldsymbol{a 2}$, Illustration showing the largest (light brown) and smallest (dark brown) spread sizes of the tracer at the delivery site. $\boldsymbol{b}$ 1, Retrogradely labeled BLA afferents were found in the LC. Scale bar, $100 \mu \mathrm{m}$. $\boldsymbol{b 2}$, A magnified view of LC. Arrowhead, c-fos only. Arrow, tracer only. ${ }^{*}$, tracer $+c$-fos. Scale bar, $50 \mu \mathrm{m} . c 1$, The number of retrogradely labeled LC neurons $[($ tracer only) + (tracer $+c$-fos) $]$ were equivalent across restraint (Res; $n=5)$ and control (NoRes; $n=4)$ conditions. c2, The number of dual-labeled neurons was significantly higher for Restraint rats than No-Restraint controls ( $\left.{ }^{*} p<0.05\right)$. c3, Acute restraint activated a significantly higher percentage of the $L C$ neurons that directly project to the BLA $\left({ }^{*} p<0.05\right)$. CeA, Central nucleus of the amygdala; $4 \mathrm{~V}$, fourth ventricle.

PRO-AMPH, $p<0.001)$. However, following amphetamine administration, there was a significantly higher locomotor activity in propranolol pretreated rats compared with saline pretreated rats (increased by 39\%; Fig. 6b2,b3, PRO-AMPH and SALAMPH, $p=0.012)$.

\section{Discussion}

In the current studies, correlated electrophysiological recordings, behavioral testing, pharmacological manipulations, and immunohistochemical approaches were used to assess the delayed response of VTA DA activity to acute restraint and how it is modulated by the noradrenergic system. Our results revealed that $24 \mathrm{~h}$ after the cessation of the $2 \mathrm{~h}$ restraint session, there was a significant decrease in VTA DA neuron population activity and a significant attenuation of amphetamine-induced locomotor activity. Moreover, systemic or intra-BLA blockade of the stressinduced increase in noradrenaline reversed the decrease in DA function. These data suggest that the noradrenergic and the dopaminergic systems interact to generate the appropriate responses when responding to a stressful event.

DA neurons exhibit burst firing when the behaving animals encounter a behaviorally salient stimulus, such as one predicting reward (Schultz, 1998). In order for burst firing to take place, the DA neuron must be in a spontaneously firing condition (Floresco et al., 2003; Sesack and Grace, 2010). Thus, the proportion of DA neurons firing spontaneously, the population activity, determines the amplitude of the DA phasic response, thus serving as an amplification factor of the salience signal (Lodge and Grace, 2006). Immediately following the acute restraint, there was a significant increase in VTA DA neuron population activity and amphetamineinduced locomotor activity (Valenti et al., 2011). Such a stress-induced increase is proposed to mimic the situation where an animal needs to attend to the salient stimulus in the environment and prepare for an appropriate goal-directed behavior. It has been suggested that acute restraintinduced changes in the DA system are persistent (Pacchioni et al., 2007). However, we found that $24 \mathrm{~h}$ after the acute restraint, there was a significant decrease in VTA DA neuron population activity, as well as a decreased behavioral response to amphetamine, which resembled those encountered in animals exposed to a chronic stressor (Moore et al., 2001). Whether this decrease is adaptive or maladaptive is open to question; however, one result of a suppressed state following a stressor would be to avoid the condition that gave rise to this stress. A context-linked decrease in DA neuron activity would be expected to attenuate a desire to be reintroduced into the stressor environment. Thus, an immediate increase in DA activity could facilitate escape, and a delayed attenuation could prevent a second occurrence of the stressor. In many ways, this is functionally analogous to the opponent process theory proposed to occur during drug exposure (Solomon and Corbit, 1974; Koob et al., 1997), in which the initial heightened response of the DA system generates an adaptive, opposing response. It is essential to follow the temporal changes in DA neuron population activity to further understand whether the decrease $24 \mathrm{~h}$ after the cessation of the acute restraint causes the animal to adaptively avoid a stressor, or alternately, is a contributing factor that leads to pathological changes in behavior or physiological responses (Cabib and Puglisi-Allegra, 1996). Although the ability of restraint to attenuate amphetamineinduced locomotion was robust across procedures, there was some inconsistency in the magnitude of the effect produced across experiments; i.e., the amphetamine response on the restraint animals is diminished in Figures 3 and 4 but is nearly abolished in Figures 2 and 6. The source of this variance at this point is unclear, and whether it was a factor related to the surgery required for cannula implantation or the stress of the systemic injection would need to be evaluated using identical testing conditions across age- and condition-matched subjects.

Stressful stimuli potently activated the noradrenergic neurons in the LC (Rasmussen et al., 1986; Aston-Jones et al., 1991; Morilak et 
al., 2005). In this study we demonstrated that prerestraint and postrestraint blockade of noradrenaline with the $\beta$-noradrenergic receptor antagonist, propranolol, successfully reversed the stress-induced decrease in VTA DA neuron population activity and attenuation in amphetamine-induced locomotor activity. The fact that postrestraint administration is effective suggests that the potentially negative response to a previously experienced stressor can be disrupted. However, propranolol given to naive animals did not increase VTA DA neuron population activity, suggesting that the $\beta$-receptor blocker is effective only when the NE levels are elevated. Thus, to effectively prevent the negative response from developing into sustained pathological long-term consequences, the intervention would have to be done in a timely manner, which is particularly critical and could be a caveat in terms of clinical application (Grissom and Bhatnagar, 2011). The effect of restraintactivated noradrenaline on VTA neurons appears to be meditated through the BLA. The LC and the BLA are reciprocally connected (Van Bockstaele et al., 2001; Buffalari and Grace, 2007), and LC neurons that project directly onto the BLA are highly activated after acute restraint. Moreover, local BLA administration of propranolol blocked the changes in VTA DA neuron population activity and the attenuated behavioral response to amphetamine. Earlier studies have demonstrated that the BLA and its modulation by norepinephrine is critically involved in the consolidation of a stressful memory (McGaugh, 2004; Roozendaal et al., 2009). Therefore, it is possible that local blockade of $\beta$-noradrenergic receptors in the BLA prevented the consolidation of the restraint experience, and thus allowed the VTA to behave normally. It is worth pointing out that different subtypes of $\beta$-receptors in the BLA exert opposing effects: $\beta 3$-receptor decreases, while $\beta 1-/$ $\beta 2$-receptors increase, local excitability (Silberman et al., 2010). Since propranolol acts primarily on $\beta 1-/ \beta 2$-receptors, it is unlikely that this effect was due to a disruption of $\beta 3$-mediated actions.

There are several potential pathways through which the BLA can modulate VTA DA activity; those of particular relevance to stress include projections to the prefrontal cortex (PFC), the hippocampus (HIPP), and the NAc (Sesack and Grace, 2010). The BLA is reciprocally connected with the PFC and the HIPP, whereas the PFC and the HIPP are also reciprocally connected (Thierry et al., 2000). All three structures have direct excitatory projections to the NAc (Thierry et al., 2000; Sesack and Grace, 2010), which then directly, and indirectly through the ventral
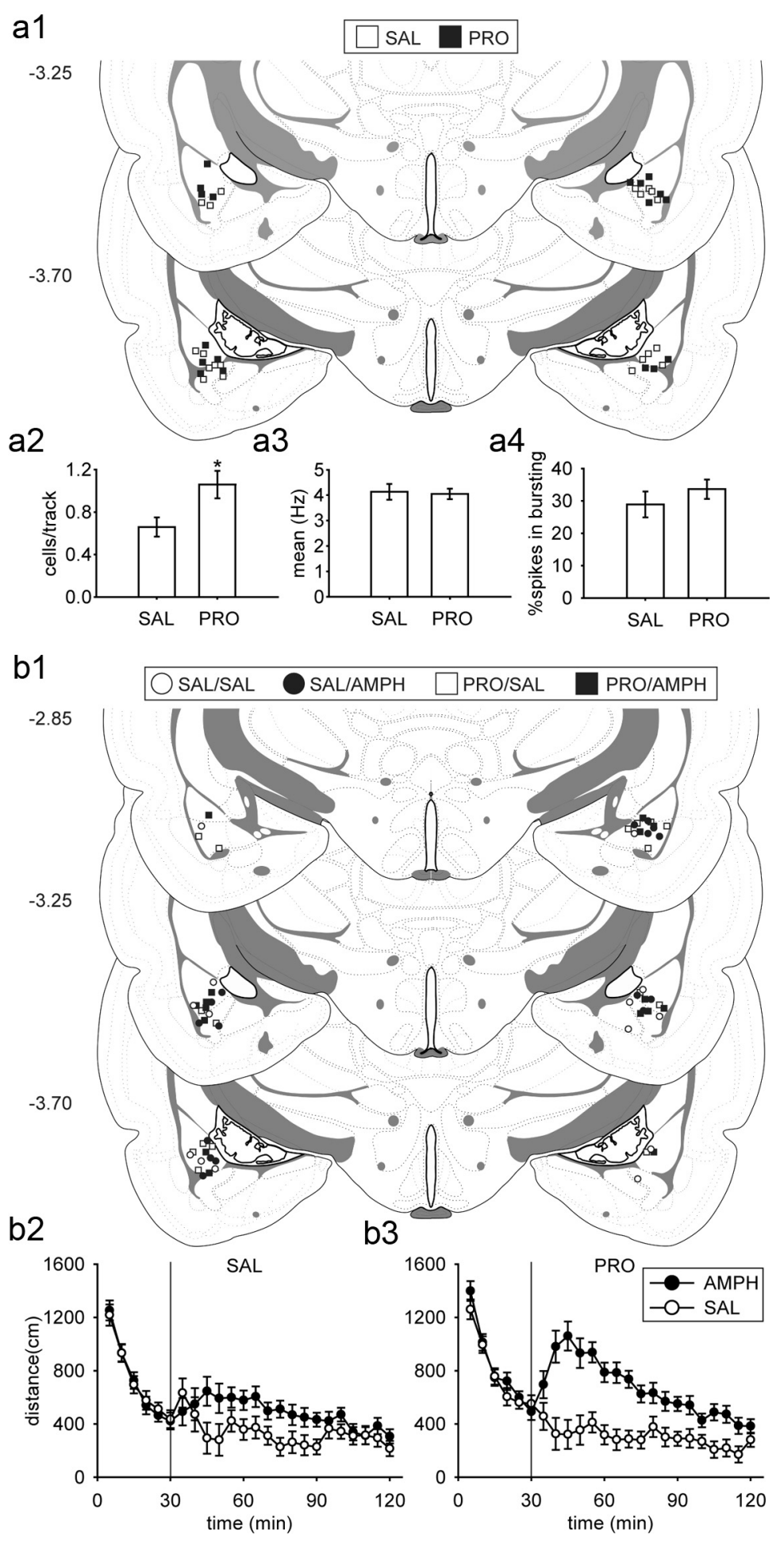

Figure 6. Prerestraint intra-BLA administration of the $\beta$-noradrenergic receptor antagonist, propranolol, blocked the decrease in VTA DA neuron population activity and reversed the attenuation of amphetamine-induced locomotor activity measured $24 \mathrm{~h}$ after restraint. $\boldsymbol{a 1}$, $\boldsymbol{b} 1$, Cannulae placements of all rats included in this study. $\boldsymbol{a} 2-\boldsymbol{a} 4$, For all rats restrained for a $2 \mathrm{~h}$ session, propranolol pretreatment (PR0; $n=9$ ) significantly increased ( $a 2)$ the DA neuron population activity than saline (SAL; $n=10)$ control $\left({ }^{*} p<0.05\right)$, with no significant difference between ( $\boldsymbol{a} 3$ ) mean firing rates or ( $\boldsymbol{a} \mathbf{4})$ bursting activity. DA neurons recorded: SAL $(n=59), \operatorname{PRO}(n=86) . \boldsymbol{b} 2, \boldsymbol{b} 3, \mathrm{AMPH}$ induced higher locomotor activity regardless of pretreatment (b2, SAL; $\boldsymbol{b} 3, \mathrm{PRO})$, with significantly higher locomotor activity in rats pretreated with PRO (all ps $<0.05$ ). Group sizes: SAL-SAL $(n=7)$, SAL-AMPH $(n=8)$, PRO-SAL $(n=8)$, and PRO-AMPH $(n=8)$.

pallidum (VP), project onto the VTA in an inhibitory manner. In addition, the amygdala projects to the rostromedial tegmental nucleus (Lavezzi and Zahm, 2011) and the VP (MaslowskiCobuzzi and Napier, 1994), both of which can inhibit the VTA. 
Therefore, the amygdala can influence VTA activity via a complex network that includes areas that can provide highly integrated information to this region. As the VTA receives integrative information from a diffused neural network, it raises the question of whether the single structure of the BLA is responsible for all of the stress-induced changes in VTA DA neuronal activity. Following the acute restraint, the immediate increase in VTA DA neuron population activity and behavioral response to amphetamine is reversed by inactivating the HIPP (Valenti et al., 2011); however, completely blocking HIPP activity does not decrease DA neuron population activity. In contrast, the decrease in VTA DA neuron population activity and behavioral response to amphetamine $24 \mathrm{~h}$ after restraint is reversed by blocking $\beta$-noradrenergic receptors in the BLA. Therefore, the HIPP and the BLA appear to exert equivalent but opposite modulatory effects on VTA DA neuron firing and the behavioral response to amphetamine.

In conclusion, the DA system is potently regulated by the integration of distinct contextual, emotional, and behaviorally salient stimuli. Moreover, the output of the DA neurons provides a critical feedback to these systems, especially the NAc, that regulate goal-directed behaviors. The balance of such multiple structures enables an organism to adapt to its environment. Conversely, when taken to an extreme, disruption of this balance can lead to maladaptive responses. By understanding the dynamics of these systems and their interactions, the ability to treat the dysregulation aroused by pathological stressors may be realized.

\section{References}

Aston-Jones G, Chiang C, Alexinsky T (1991) Discharge of noradrenergic locus coeruleus neurons in behaving rats and monkeys suggests a role in vigilance. Prog Brain Res 88:501-520. CrossRef Medline

Bienkowski MS, Rinaman L (2011) Immune challenge activates neural inputs to the ventrolateral bed nucleus of the stria terminalis. Physiol Behav 104:257-265. CrossRef Medline

Buffalari DM, Grace AA (2007) Noradrenergic modulation of basolateral amygdala neuronal activity: opposing influences of alpha- 2 and beta receptor activation. J Neurosci 27:12358-12366. CrossRef Medline

Cabib S, Puglisi-Allegra S (1996) Stress, depression and the mesolimbic dopamine system. Psychopharmacology 128:331-342. CrossRef Medline

Cifelli P, Grace AA (2012) Pilocarpine-induced temporal lobe epilepsy in the rat is associated with increased dopamine neuron activity. Int J Neuropsychopharmacol 15:957-964. CrossRef Medline

Condé F, Maire-Lepoivre E, Audinat E, Crépel F (1995) Afferent connections of the medial frontal cortex of the rat. II. Cortical and subcortical afferents. J Comp Neurol 352:567-593. CrossRef Medline

Dębiec J, Bush DE, LeDoux JE (2011) Noradrenergic enhancement of reconsolidation in the amygdala impairs extinction of conditioned fear in rats-a possible mechanism for the persistence of traumatic memories in PTSD. Depress Anxiety 28:186-193. CrossRef Medline

de Kloet ER, Joëls M, Holsboer F (2005) Stress and the brain: from adaptation to disease. Nat Rev Neurosci 6:463-475. CrossRef Medline

Floresco SB, West AR, Ash B, Moore H, Grace AA (2003) Afferent modulation of dopamine neuron firing differentially regulates tonic and phasic dopamine transmission. Nat Neurosci 6:968-973. CrossRef Medline

Goebel M, Stengel A, Wang L, Taché Y (2009) Restraint stress activates nesfatin-1-immunoreactive brain nuclei in rats. Brain Res 1300:114-124. CrossRef Medline

Grace AA, Bunney BS (1983) Intracellular and extracellular electrophysiology of nigral dopaminergic neurons-1. Identification and characterization. Neuroscience 10:301-315. CrossRef Medline

Grace AA, Bunney BS (1984) The control of firing pattern in nigral dopamine neurons: burst firing. J Neurosci 4:2877-2890. Medline

Grace AA, Floresco SB, Goto Y, Lodge DJ (2007) Regulation of firing of dopaminergic neurons and control of goal-directed behaviors. Trends Neurosci 30:220-227. CrossRef Medline

Grissom NM, Bhatnagar S (2011) The basolateral amygdala regulates adaptation to stress via beta-adrenergic receptor-mediated reductions in phosphorylated extracellular signal-regulated kinase. Neuroscience 178:108-122. CrossRef Medline
Herman JP, Cullinan WE (1997) Neurocircuitry of stress: central control of the hypothalamo-pituitary-adrenocortical axis. Trends Neurosci 20:78-84. CrossRef Medline

Herry C, Ciocchi S, Senn V, Demmou L, Müller C, Lüthi A (2008) Switching on and off fear by distinct neuronal circuits. Nature 454:600-606. CrossRef Medline

Joëls M, Baram TZ (2009) The neuro-symphony of stress. Nat Rev Neurosci 10:459-466. Medline

Johnson LR, Hou M, Prager EM, Ledoux JE (2011) Regulation of the fear network by mediators of stress: norepinephrine alters the balance between cortical and subcortical afferent excitation of the lateral amygdala. Front Behav Neurosci 5:23. Medline

Jones BE, Moore RY (1977) Ascending projections of the locus coeruleus in the rat. II. Autoradiographic study. Brain Res 127:25-53. Medline

Knapska E, Maren S (2009) Reciprocal patterns of c-Fos expression in the medial prefrontal cortex and amygdala after extinction and renewal of conditioned fear. Learn Mem 16:486-493. CrossRef Medline

Koob GF, Caine SB, Parsons L, Markou A, Weiss F (1997) Opponent process model and psychostimulant addiction. Pharmacol Biochem Behav 57:513-521. CrossRef Medline

Lavezzi HN, Zahm DS (2011) The mesopontine rostromedial tegmental nucleus: an integrative modulator of the reward system. Basal Ganglia 1:191-200. CrossRef Medline

LeDoux JE (2000) Emotion Circuits in the Brain. Annu Rev Neurosci 23: 155-184. CrossRef Medline

Lodge DJ, Grace AA (2006) The hippocampus modulates dopamine neuron responsivity by regulating the intensity of phasic neuron activation. Neuropsychopharmacology 31:1356-1361. CrossRef Medline

Lodge DJ, Grace AA (2007) Aberrant hippocampal activity underlies the dopamine dysregulation in an animal model of schizophrenia. J Neurosci 27:11424-11430. CrossRef Medline

Lodge DJ, Grace AA (2008) Amphetamine activation of hippocampal drive of mesolimbic dopamine neurons: a mechanism of behavioral sensitization. J Neurosci 28:7876-7882. CrossRef Medline

Luo AH, Georges FE, Aston-Jones GS (2008) Novel neurons in ventral tegmental area fire selectively during the active phase of the diurnal cycle. Eur J Neurosci 27:408-422. CrossRef Medline

Maslowski-Cobuzzi RJ, Napier TC (1994) Activation of dopaminergic neurons modulates ventral pallidal responses evoked by amygdala stimulation. Neuroscience 62:1103-1119. CrossRef Medline

Mcdonald AJ, Mascagni F, Guo L (1996) Projections of the medial and lateral prefrontal cortices to the amygdala: a Phaseolus vulgaris leucoagglutinin study in the rat. Neuroscience 71:55-75. CrossRef Medline

McEwen BS (2007) Physiology and neurobiology of stress and adaptation: central role of the brain. Physiol Rev 87:873-904. CrossRef Medline

McGaugh JL (2004) The amygdala modulates the consolidation of memories of emotionally arousing experiences. Annu Rev Neurosci 27:1-28. CrossRef Medline

Moore H, Rose HJ, Grace AA (2001) Chronic cold stress reduces the spontaneous activity of ventral tegmental dopamine neurons. Neuropsychopharmacology 24:410-419. CrossRef Medline

Morilak DA, Barrera G, Echevarria DJ, Garcia AS, Hernandez A, Ma S, Petre CO (2005) Role of brain norepinephrine in the behavioral response to stress. Prog Neuropsychopharmacol Biol Psychiatry 29:1214-1224. CrossRef Medline

Pacchioni AM, Cador M, Bregonzio C, Cancela LM (2007) A glutamatedopamine interaction in the persistent enhanced response to amphetamine in nucleus accumbens core but not shell following a single restraint stress. Neuropsychopharmacology 32:682-692. CrossRef Medline

Pitkänen A, Pikkarainen M, Nurminen N, Ylinen A (2000) Reciprocal connections between the amygdala and the hippocampal formation, perirhinal cortex, and postrhinal cortex in rat. A review. Ann N Y Acad Sci 911:369-391. Medline

Rasmussen K, Morilak DA, Jacobs BL (1986) Single unit activity of locus coeruleus neurons in the freely moving cat. I. During naturalistic behaviors and in response to simple and complex stimuli. Brain Res 371:324334. CrossRef Medline

Roozendaal B, Okuda S, Van der Zee EA, McGaugh JL (2006) Glucocorticoid enhancement of memory requires arousal-induced noradrenergic activation in the basolateral amygdala. Proc Natl Acad Sci U S A 103: 6741-6746. CrossRef Medline

Roozendaal B, Castello NA, Vedana G, Barsegyan A, McGaugh JL (2008) Nor- 
adrenergic activation of the basolateral amygdala modulates consolidation of object recognition memory. Neurobiol Learn Mem 90:576-579. CrossRef Medline

Roozendaal B, McEwen BS, Chattarji S (2009) Stress, memory and the amygdala. Nat Rev Neurosci 10:423-433. CrossRef Medline

Salmon P, Stanford SC (1989) Beta-adrenoceptor binding correlates with behaviour of rats in the open field. Psychopharmacology 98:412-416. CrossRef Medline

Sawchenko PE, Li HY, Ericsson A (2000) Circuits and mechanisms governing hypothalamic responses to stress: a tale of two paradigms. Prog Brain Res 122:61-78. Medline

Schultz W (1998) Predictive reward signal of dopamine neurons. J Neurophysiol 80:1-27. Medline

Sesack SR, Grace AA (2010) Cortico-Basal Ganglia reward network: microcircuitry. Neuropsychopharmacology 35:27-47. CrossRef Medline

Silberman Y, Ariwodola OJ, Chappell AM, Yorgason JT, Weiner JL (2010) Lateral paracapsular GABAergic synapses in the basolateral amygdala contribute to the anxiolytic effects of $\beta 3$ adrenoceptor activation. Neuropsychopharmacology 35:1886-1896. CrossRef Medline

Solomon RL, Corbit JD (1974) An opponent-process theory of motivation. I. Temporal dynamics of affect. Psychol Rev 81:119-145. CrossRef Medline
Thierry AM, Gioanni Y, Dégénétais E, Glowinski J (2000) Hippocampoprefrontal cortex pathway: anatomical and electrophysiological characteristics. Hippocampus 10:411-419. CrossRef Medline

Tully K, Bolshakov VY (2010) Emotional enhancement of memory: how norepinephrine enables synaptic plasticity. Mol Brain 3:15. CrossRef Medline

Ulrich-Lai YM, Herman JP (2009) Neural regulation of endocrine and autonomic stress responses. Nat Rev Neurosci 10:397-409. CrossRef Medline

Ungless MA, Grace AA (2012) Are you or aren't you? Challenges associated with physiologically identifying dopamine neurons. Trends Neurosci 35 : 422-430. CrossRef Medline

Valenti O, Lodge DJ, Grace AA (2011) Aversive stimuli alter ventral tegmental area dopamine neuron activity via a common action in the ventral hippocampus. J Neurosci 31:4280-4289. CrossRef Medline

Van Bockstaele EJ, Bajic D, Proudfit H, Valentino RJ (2001) Topographic architecture of stress-related pathways targeting the noradrenergic locus coeruleus. Physiol Behav 73:273-283. CrossRef Medline

Zimmerman JM, Maren S (2010) NMDA receptor antagonism in the basolateral but not central amygdala blocks the extinction of Pavlovian fear conditioning in rats. Eur J Neurosci 31:1664-1670. Medline 